Supporting Information for:

\title{
Complex Movements in Rotaxanes: Shuttling Coupled with Conformational Transition of Cyclodextrins
}

Shuangshuang Wang, ${ }^{a}$ Tanfeng Zhao, ${ }^{a}$ Xueguang Shao, ${ }^{\text {a,b,c,d }}$ Christophe Chipot ${ }^{\mathrm{e}, \mathrm{f,g}}$ and Wensheng Cai ${ }^{*, \mathrm{a}, \mathrm{b}, \mathrm{d}}$

${ }^{a}$ Research Center for Analytical Sciences, College of Chemistry, Nankai University, Tianjin, 300071, China

${ }^{b}$ Tianjin Key Laboratory of Biosensing and Molecular Recognition, Tianjin, 300071, China

${ }^{c}$ State Key Laboratory of Medicinal Chemical Biology, Tianjin, 300071, China

${ }^{d}$ Collaborative Innovation Center of Chemical Science and Engineering, Tianjin, 300071, China

${ }^{\mathrm{e}}$ Laboratoire International Associé Centre National de la Recherche Scientifique et University of Illinois at Urbana-Champaign, Unité Mixte de Recherche No. 7565, Université de Lorraine, B.P. 70239, 54506

Vandoeuvre-lès-Nancy cedex, France

${ }^{f}$ Theoretical and Computational Biophysics Group, Beckman Institute, University of Illinois at Urbana-Champaign, Urbana, Illinois 61801, United States

${ }^{g}$ Department of Physics, University of Illinois at Urbana-Champaign, Urbana, Illinois 61801, United States

* To whom correspondence should be addressed. E-mail: wscai@nankai.edu.cn (W.C). 
Validation of the Collective Variable $\varphi$. From the trajectories of the one-dimensional (1D) ABF simulations, it was observed that several GLUs underwent isomerization during shuttling. However, these isomerization processes are not independent. In addition, for practical reasons, the dimensionality in the ABF method cannot go much beyond than three. In the present study, only the isomerization of one GLU in CDs is described, and the conformational change of this GLU is expected to drive other GLUs to transform their conformation accordingly. In the 2D free-energy calculations for the shuttling coupled with isomerization, the transition coordinate consists of the distance $\xi$ and the endocyclic dihedral angle $\varphi$ (see Scheme 1 and 2 in the manuscript). To validate the collective variable $\varphi$, the 1D free-energy profile characterizing the conformational transition from ${ }^{4} \mathrm{C}_{1}$ to ${ }^{1} \mathrm{C}_{4}$ of a GLU in vacuum using $\varphi$ as the model reaction coordinate is determined (see Figure S1). Analysis of the ABF simulation trajectory shows that the structures at the free-energy minimums, $\varphi=+60^{\circ}$, and $-60^{\circ}$, correspond to the ${ }^{4} \mathrm{C}_{1}$ and ${ }^{1} \mathrm{C}_{4}$ conformation, respectively, and the structure at the maximum, $\varphi=0^{\circ}$, corresponds to a mixture of several kinds of conformations, such as $\mathrm{B}_{\mathrm{O}, 3},{ }^{4} \mathrm{H}_{5}$, and ${ }^{\mathrm{O}, 3} \mathrm{~B}$, wherein the main component is the $\mathrm{B}_{\mathrm{O}, 3}$ one. The free-energy difference between the ${ }^{1} \mathrm{C}_{4}$ and the ${ }^{4} \mathrm{C}_{1}$ conformations is estimated to be $6.0 \mathrm{kcal} / \mathrm{mol}$, which agrees well with that obtained by theoretical calculations, being about $5.5 \mathrm{kcal} / \mathrm{mol}$, reported in previous literatures. ${ }^{1,2}$ The free-energy barrier with respect to the stable state corresponding the ${ }^{4} \mathrm{C}_{1}$ conformation is calculated to be $11.1 \mathrm{kcal} / \mathrm{mol}$, in line with the free-energy difference between the $\mathrm{B}_{\mathrm{O}, 3}$ and ${ }^{4} \mathrm{C}_{1}$ conformations, being about $11.3 \mathrm{kcal} / \mathrm{mol}$. Therefore, though only using $\varphi$ cannot describe precisely all the transition states during the transition process, it is still feasible to use this variable to approximately characterize the isomerization of the GLU. 


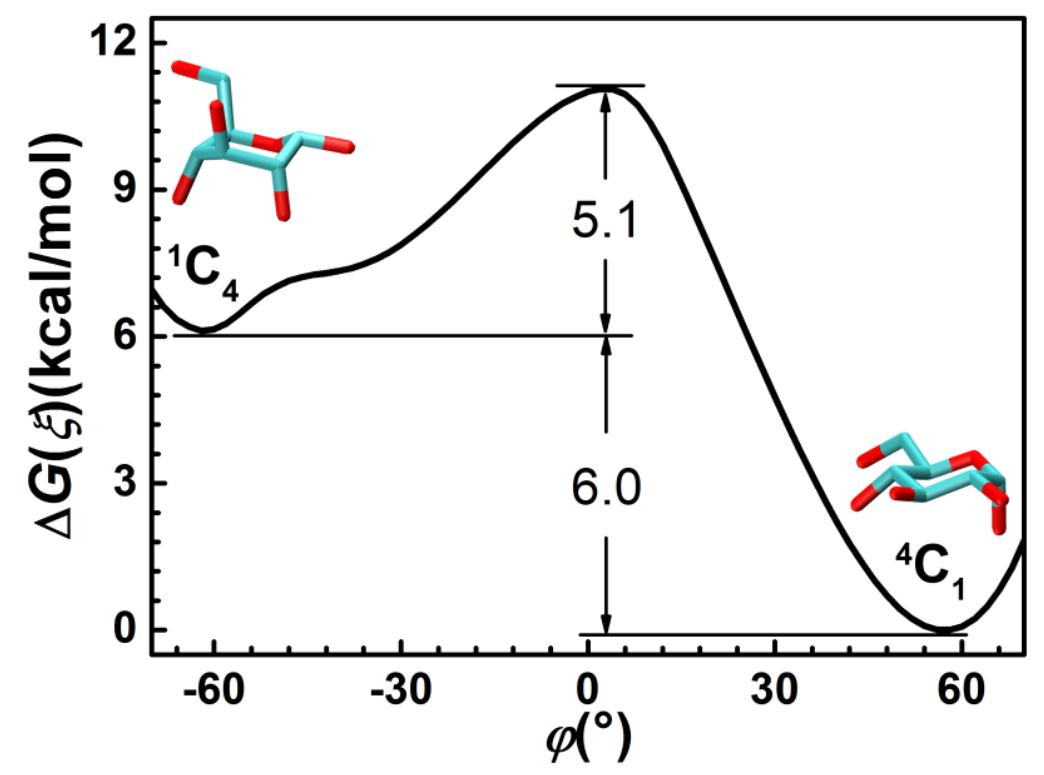

Figure S1. 1D free-energy profile characterizing the conformational transition of a GLU from ${ }^{4} \mathrm{C}_{1}$ to ${ }^{1} \mathrm{C}_{4}$ conformation in vacuum.
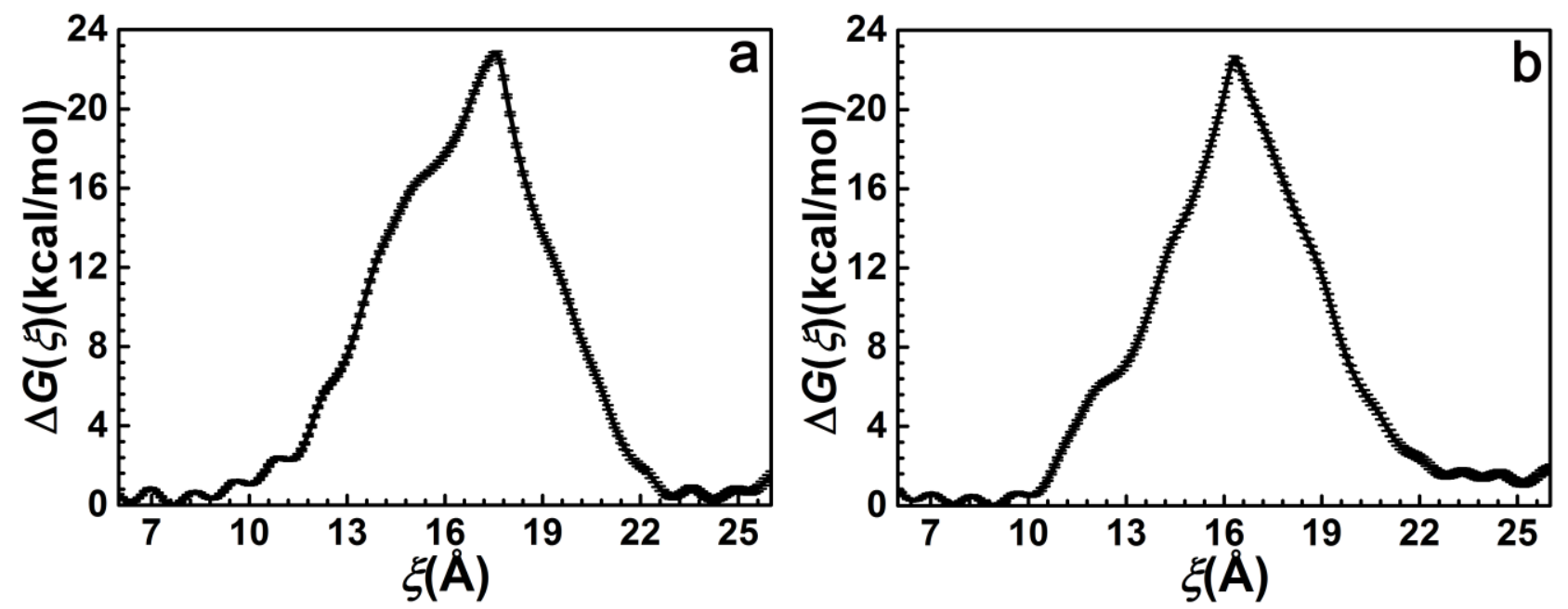

Figure S2. 1D free-energy profiles of rotaxane 1A (a) and 1B (b) featuring the shuttling process of the $\alpha$-CD along $\xi$. The results show that the free-energy differences do not match the experimental measurements. Thus, 1D free-energy calculations are not suitable for delineating the shuttling process of rotaxane $1 \mathrm{~A}$ and $1 \mathrm{~B}$. 

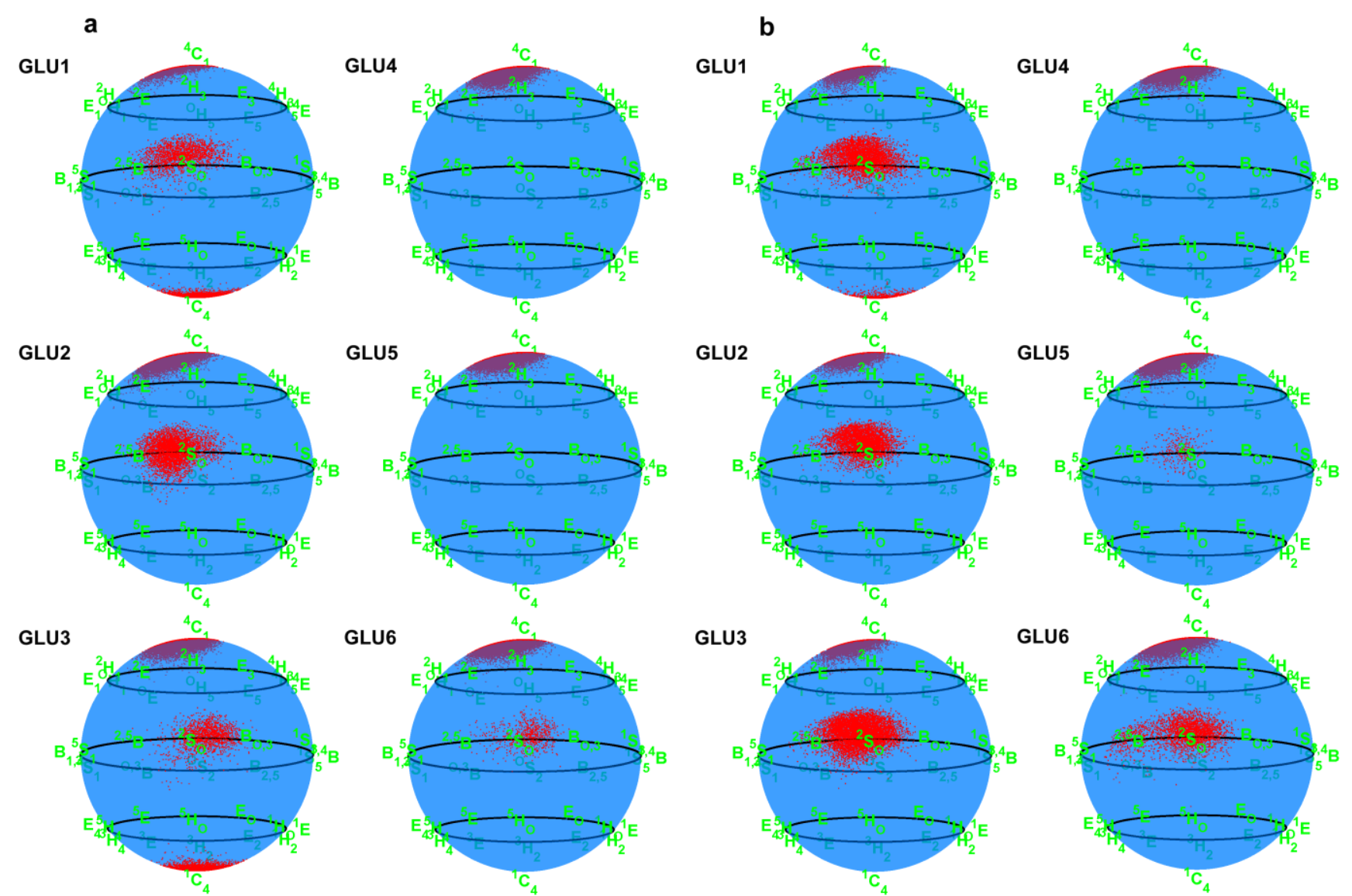

Figure S3. Distribution of the puckering conformations of each GLU in the $\alpha$-CD, in the region corresponding to the free-energy mimima and maximum along the least free-energy pathway in Figure 1, on the Cremer-Pople sphere, using the spherical coordinate system $(Q, \theta, \phi)$ defined by Cremer and Pople. ${ }^{3} Q$ is the total puckering amplitude. Here $Q$ is a constant. (a) Rotaxane $1 \mathrm{~A}$, (b) Rotaxane $1 \mathrm{~B}$. The results indicate that (i) four GLUs in rotaxane 1A, and (ii) five GLUs in rotaxane 1B, underwent interconversion between three conformations ${ }^{4} \mathrm{C}_{1}\left(\theta=0^{\circ}, \phi=0-360^{\circ}\right),{ }^{2} \mathrm{~S}_{\mathrm{O}}\left(\theta=90^{\circ}, \phi=150^{\circ}\right)$ and ${ }^{1} \mathrm{C}_{4}(\theta$ $\left.=180^{\circ}, \phi=0-360^{\circ}\right)$.

\section{REFERENCES:}

(1) Appell, M.; Strati, G.; Willett, J. L.; Momany, F. A. B3LYP/6-311++G** study of $\alpha-$ and $\beta$-Dglucopyranose and 1,5-anhydro-D-glucitol: ${ }^{4} \mathrm{C}_{1}$ and ${ }^{1} \mathrm{C}_{4}$ chairs, ${ }^{(3,0)} \mathrm{B}$ and $\mathrm{B}_{(3,0)}$ boats, and skew-boat conformations. Carbohydr. Res. 2004, 339, 537-551 
(2) Mayes, H. B.; Broadbelt, L. J.; Beckham, G. T. How sugars pucker: electronic structure calculations map the kinetic landscape of five biologically paramount monosaccharides and their implications for enzymatic catalysis. J. Am. Chem. Soc. 2014, 136, 1008-1022.

(3) Cremer, D.; Pople, J. A. General definition of ring puckering coordinates. J. Am. Chem. Soc. 1975, 97, 1354-1358. 